\title{
Fractonic superfluids. II. Condensing subdimensional particles
}

\author{
Shuai A. Chen, ${ }^{1}$ Jian-Keng Yuan, ${ }^{2}$ and Peng Ye ${ }^{2, *}$ \\ ${ }^{1}$ Institute for Advanced Study, Tsinghua University, Beijing 100084, China \\ ${ }^{2}$ School of Physics and State Key Laboratory of Optoelectronic Materials and Technologies, \\ Sun Yat-sen University, Guangzhou 510275, China
}

(Received 29 October 2020; revised 20 January 2021; accepted 16 February 2021; published 9 March 2021; corrected 7 April 2021)

\begin{abstract}
As a series of work about "fractonic superfluids," in this paper, we develop an exotic fractonic superfluid phase in $d$-dimensional space where subdimensional particles-their mobility is partially restricted-are condensed. The off-diagonal long range order (ODLRO) is investigated. To demonstrate, we consider "lineons"-a subdimensional particle whose mobility is free only in certain one-dimensional directions. We start with a $d$-component microscopic Hamiltonian model. The model respects a higher-rank symmetry such that both particle numbers of each component and angular charge moments are conserved quantities. By performing the Hartree-Fock-Bogoliubov approximation, we derive a set of Gross-Pitaevskii equations and a Bogoliubov-de Gennes (BdG) Hamiltonian, which leads to a description of both condensed components and unification of gapless phonons and gapped rotons. With the coherent-path-integral representation, we also derive the longwavelength effective field theory of gapless Goldstone modes and analyze quantum fluctuations around classical ground states. The Euler-Lagrange equations and Noether charges/currents are also studied. In two spatial dimensions and higher, such an ODLRO stays stable against quantum fluctuations. Finally, we study vortex configurations. The higher-rank symmetry enforces a hierarchy of thermal vortex excitations whose structures are dominated by two guiding statements. Specially, we construct two types of vortex excitations, the conventional and dipole vortices. The latter carries a charge with dimension as a momentum. The two statements can be more generally applicable. Further perspectives are discussed.
\end{abstract}

DOI: 10.1103/PhysRevResearch.3.013226

\section{INTRODUCTION}

As exotic states of matter, fracton topological order can be characterized by noise-immune ground state degeneracy that unconventionally depends on the system size on a nontrivial compact manifold [1-4]. Recently, fracton topological order or fracton physics in a more general sense has been intensively investigated; see, e.g., Refs. [1-62]. A recent review can be found in Refs. [63,64]. Topological excitations of fracton topological order include fractons, subdimensional particles $[1-4,26]$, and more complicated spatially extended excitations [27]. One of the remarkable features of these excitations is topological restriction on mobility: Their geometrical locations cannot be freely changed by any local operators. More concretely, mobility of fractons is completely frozen while subdimensional particles are still allowed to move but within a certain cluster of lower-dimensional subspace. As two examples of subdimensional particles in the $X$-cube lattice model [1-4], lineons and planeons can move along certain one-dimensional directions and two-dimensional parallel

\footnotetext{
*yepeng5@mail.sysu.edu.cn

Published by the American Physical Society under the terms of the Creative Commons Attribution 4.0 International license. Further distribution of this work must maintain attribution to the author(s) and the published article's title, journal citation, and DOI.
}

planes, respectively. Instead of the interpretation as "topological excitations," one can also regard all these strange particles, i.e., fractons, lineons, and planeons as original bosons, which leads to unconventional many-body physics. In this context, the restriction on mobility is ascribed to the implementation of so-called "higher-rank symmetry." The latter guarantees a set of higher moments are conserved $[25,33,65]$.

In a previous work [47] of many-body physics of fractons, the authors of the present work proposed a fractonic superfluid phase formed by nonrelativistic bosonic fractons in $d$ spatial dimensions $(d \mathrm{D})$. The phase can be regarded as a result of spontaneous breakdown of higher-rank symmetry. Due to a higher-rank symmetry, both total dipole moments and the total particle number (charge) are conserved. The microscopic Hamiltonian that respects such a symmetry must be non-Gaussian, which naturally forbids a single fracton from freely propagating. Starting with the first-order time derivative just like a conventional superfluid phase, we add a usual Mexican-hat potential for fractons. When the chemical potential is turned from a negative to positive value, the system undergoes a quantum phase transition from the normal state to the superfluid phase. The latter is manifested by occupation of a macroscopic number of fractons on the same quantum state, which leads to the formation of an off-diagonal long range order (ODLRO) [66]. As a direct consequence of non-Gaussianality, the corresponding Euler-Lagrange equation is highly nonlinear, from which one can extract the 
TABLE I. Comparison between a conventional superfluid phase (denoted as $d \mathrm{SF}^{d}$ ), fractonic superfluid phase (denoted as $d \mathrm{SF}^{0}$; see Ref. [47]) via condensing fractons, and a fractonic superfluid phase (denoted as $d S^{1}$ ) via condensing lineons. In these three types of superfluids, the condensed particles are, respectively, usual bosons of full mobility, fractons without any mobility, and lineons with partial mobility. Vortex excitations in $2 \mathrm{D}$ form a hierarchy where $\ell, \ell_{1}, \ell_{2}$ denote winding numbers and $p, p_{1}, p_{2}$ are quantized as momenta with $\varphi(\mathbf{x})$ being the relative angle of site $\mathbf{x}$ to the vortex core.

\begin{tabular}{|c|c|c|c|}
\hline & $d \mathrm{SF}^{d}$ & $d \mathrm{SF}^{0}$ & $d \mathrm{SF}^{1}$ \\
\hline Conserved quantities & Total charge & Total charge, total dipole moment & Total charges, angular charge moments \\
\hline Order parameter & $\sqrt{\rho_{0}} e^{i \theta_{0}}$ & $\sqrt{\rho_{0}} e^{i\left(\theta_{0}+\sum_{a} \beta_{a} x^{a}\right)}$ & $\sqrt{\rho_{0}} e^{i\left(\theta_{a}+\sum_{b} \beta_{a b} x^{a}\right)}\left(\beta_{a b}=-\beta_{b a}\right)$ \\
\hline Plane-wave dispersion & Dispersive & Dispersionless & Partially dispersive \\
\hline Ground state & $e^{\int d^{d} x \sqrt{\rho_{0}} e^{i \theta_{0}} \hat{\Phi}^{\dagger}(\mathbf{x})}|0\rangle$ & $e^{\int d^{d} x \sqrt{\rho_{0}} e^{i\left(\theta_{0}+\sum_{a} \beta_{a} x^{a}\right)} \hat{\Phi}^{\dagger}(\mathbf{x})}|0\rangle$ & $\prod_{a} e^{\int d^{d} x \sqrt{\rho_{0}} e^{i\left(\theta_{a}+\sum_{b} \beta_{a b} x^{a}\right)} \hat{\Phi}_{a}^{\dagger}(\mathbf{x})}|0\rangle$ \\
\hline Specific capacity heat & $c_{v} \propto T^{d}$ & $c_{v} \propto T^{\frac{d}{2}}$ & $c_{v} \propto T^{d}$ \\
\hline Number of Goldstone modes & 1 & 1 & $d$ \\
\hline Dispersion of Goldstone modes & $\omega \propto|\mathbf{k}|$ & $\omega \propto|\mathbf{k}|^{2}$ & $\omega \propto|\mathbf{k}|$ \\
\hline Stable dimension at $T=0$ & $d>1$ & $d>2$ & $d>1$ \\
\hline Vortex structure in $d=2$ & $\ell \varphi(\mathbf{x})$ & $\ell \varphi(\mathbf{x}),\left(p_{1} x+p_{2} y\right) \varphi(\mathbf{x})$ & $\begin{array}{l}\ell_{1} \varphi(\mathbf{x}), p x^{2} \varphi(\mathbf{x}) \\
\ell_{2} \varphi(\mathbf{x}), p x^{1} \varphi(\mathbf{x})\end{array}$ \\
\hline
\end{tabular}

time-dependent Gross-Pitaevskii equation that governs hydrodynamical behaviors. Furthermore, by taking quantum phase fluctuations into consideration, we find that ODLRO keeps stable in spatial dimensions $d>2$. In one dimension, the correlation function of the superfluid order parameter exponentially decays at long distances. In two dimensions (2D), it decays in a power-law pattern at zero temperature.

As mentioned above, fractons are just one of many strange particles proposed in fracton topological order. We expect that subdimensional particles can form even more exotic phases of matter as their mobility is partially rather than completely restricted. Along this line, in this work, we consider more general variants of fractonic superfluids where, instead of completely immobile fractons, subdimensional particles meet Mexican hats and thus form a superfluid. For convenience, we introduce a notation $d \mathrm{SF}^{n}$ ("SF" stands for "superfluid") which represents a superfluid phase in $d$ spatial dimensions via condensing subdimensional particles of dimension $n(0 \leqslant$ $n \leqslant d)$. For example, $d \mathrm{SF}^{d}$ denotes a conventional superfluid where the bosons are free to move in the whole space and the fractonic superfluid phase in a many-fracton model [47] is symbolized as $d \mathrm{SF}^{0}$.

More specifically, in this work, we take lineons as an example, which leads to a fractonic superfluid phase denoted by $d \mathrm{SF}^{1}$. The corresponding microscopic second-quantized Hamiltonian model $\mathcal{H}$ contains $d$ components of bosonic lineons. The Hamiltonian has strongly anistropic quadratic Gaussian terms such that mobility restriction of lineons is correctly encoded. Meanwhile, both angular charge moments and particle numbers of each component are conserved due to the presence of quartic terms that respect a higher-rank symmetry. The candidate Hamiltonian is referred to in Ref. [25] with the second time-derivative terms. Distinguishably, we set about the first-time derivative and apply a Mexican-hat potential to each component. In the coherent-path-integral representation, the Hamiltonian density $\mathcal{H}$ is sent to the Lagrangian density $\mathcal{L}=\sum_{a=1}^{d} i \phi_{a}^{*} \partial_{t} \phi_{a}-\mathcal{H}$ after a Wick rotation. Due to the firstorder time derivative, we are legitimate to interpret $\phi_{a}^{*} \phi_{a}$ as the particle density of the $a^{\text {th }}$ component, which is common in condensed matter and cold-atom systems.
When the chemical potential is turned to a positive value from a negative one, a quantum phase transition occurs from the normal state to the fractonic superfluid phase $d \mathrm{SF}^{1}$. The normal state has a unique ground state with vanishing momentum. Instead, in $d \mathrm{SF}^{1}$, ODLRO the ground states are macroscopically degenerate in the classical level and their configurations appear to be a plane wave with a finite momentum and a finite density distribution which establishes a ODLRO. Upon the Hartree-Fock-Bogoliubov mean-field approximation is applied, the boson fields are further split into two components - the normal and the condensed components, towards which we derive a set of nonlinear Bogoliubov-de Gennes equations and Gross-Pitaevskii Hamiltonian, respectively. To deal with gapless Goldstone modes and quantum phase fluctuations (i.e., ODLRO stability at infrared limit), we turn to the framework of an effective field theory. In contrast to the non-Gaussian system in Ref. [47], existence of spatially anisotropic Gaussianality ensures that a superfluid phase or ODLRO can survive against quantum fluctuations in spatial dimensions $d>1$ at zero temperature and thus this model appears more tractable experimentally. For vortex configurations in $2 \mathrm{SF}^{1}$, we point out and apply two statements for the purpose of constructing vortex configurations. The first statement dominates the multivalued part to meet the single valueness of vortex fields and the second controls the smooth part to satisfy a relation between representations of a higher-rank group and a particle number conservation symmetry group. The two statements lead to two types of vortices: the conventional vortex and dipole vortex. The latter carries a dipole charge that is quantized like a momentum. It can be detected by a vorticity from recombination between Noether currents. In fact, the two statements can be applied to point vortex excitations with a general higher-rank symmetry. In Table I, we compare different properties of a conventional superfluid phase $2 \mathrm{SF}^{2}$, fractonic superfluid via condensing fractons $2 S^{0}$ in Ref. [47] and fractonic superfluid by condensing lineons $2 \mathrm{SF}^{1}$ in this work.

The remaining part of this paper is organized as follows. Section II provides a microscopic multicomponent model and Hartree-Fork-Bogoliubov treatment. An effective field theoretical analysis is performed in Sec. III. In Sec. IV, exotic 
superfluid vortices are studied. This work is concluded in Sec. V.

\section{MICROSCOPIC SYSTEM AND MEAN-FIELD THEORY}

In this section, we start with a microscopic model of lineons, which is formulated in the second quantization language with conserved angular charge moments. This conservation is vital to the mobility restriction of lineons. Under the circumstance of condensing lineons, we apply the HartreeFock-Bogoliubov (HFB) mean-field theory to derive the Gross-Pitaevskii (GP) equations and Bogoliubov-de Gennes (BdG) Hamiltonian [67]. The former govern the order parameter of the superfluid phase and the latter unifies both gapless phonon and gapped roton modes.

\section{A. A model Hamiltonian}

In condensed matter systems, strong anisotropy can constrain particle's propagation. For example, divergence of effective mass localizes particle spatially and a strong electric field allows charged particles to move exclusively along the direction of electric field. We have investigated one microscopic realization of fractons with fully restricted motion in Ref. [47]. As a series of works, here we focus on $d$ component fields $\hat{\Phi}=\left(\hat{\Phi}_{1}, \cdots, \hat{\Phi}_{d}\right)$ in $d$ spatial dimensions in the Hamiltonian $H=\int d^{d} \mathbf{x} \mathcal{H}$ where Hamiltonian density $\mathcal{H}$ reads

$$
\begin{aligned}
\mathcal{H}= & \sum_{a=1}^{d} \partial_{a} \hat{\Phi}_{a}^{\dagger} \partial_{a} \hat{\Phi}_{a}+\sum_{a \neq b}^{d} \frac{1}{2} K_{a b}\left(\hat{\Phi}_{a}^{\dagger} \partial_{a} \hat{\Phi}_{b}^{\dagger}+\hat{\Phi}_{b}^{\dagger} \partial_{b} \hat{\Phi}_{a}^{\dagger}\right) \\
& \times\left(\hat{\Phi}_{a} \partial_{a} \hat{\Phi}_{b}+\hat{\Phi}_{b} \partial_{b} \hat{\Phi}_{a}\right)+V\left(\hat{\Phi}^{\dagger}, \hat{\Phi}\right) .
\end{aligned}
$$

The complex fields $\hat{\Phi}_{a}^{\dagger}(\mathbf{x})$ and $\hat{\Phi}_{b}(\mathbf{x})$ create and annihilate an $a$ th-component particle, respectively, and satisfy the bosonic communication relations,

$$
\left[\hat{\Phi}_{a}(\mathbf{x}), \hat{\Phi}_{b}^{\dagger}(\mathbf{y})\right]=\delta_{a b} \delta^{d}(\mathbf{x}-\mathbf{y}),
$$

where $\mathbf{x}=\left(x^{1}, \ldots, x^{d}\right)$ is the spatial coordinate. At the quadratic level, each component can only propagate in one certain spatial direction and we set the mass before the quadratic terms to be a unit. For convenience, one can set diagonal terms $K_{a a}=0$ for $a=1, \ldots, d$ since diagonal terms $K_{a a}$ is absent in Eq. (1). For simplicity, we can take the term $V\left(\hat{\Phi}^{\dagger}, \hat{\Phi}\right)$ to be the Mexican-hat potential with componentindependent chemical potential $\mu$ and interaction coupling constant $g>0$,

$$
V\left(\hat{\Phi}^{\dagger}, \hat{\Phi}\right)=\sum_{a=1}^{d}-\mu \hat{\Phi}_{a}^{\dagger} \hat{\Phi}_{a}+\frac{g}{2} \hat{\Phi}_{a}^{\dagger} \hat{\Phi}_{a}^{\dagger} \hat{\Phi}_{a} \hat{\Phi}_{a},
$$

which describes a short-range repulsive interaction via the $s$-wave scattering. In the following, no Einstein summation rule is assumed. The Hamiltonian in Eq. (1) conserves both particle numbers of each component $Q_{a} \equiv \int d^{d} x \hat{\rho}_{a}$ and angular charge moment $Q_{a b}=\int d x^{d}\left(\hat{\rho}_{a} x^{b}-\hat{\rho}_{b} x^{a}\right)$ with $\hat{\rho}_{a}=$ $\hat{\Phi}_{a}^{\dagger} \hat{\Phi}_{a}$ being the number operator of ath particles. Accordingly, the symmetry group is composed of transformations $\hat{\Phi}_{a} \rightarrow e^{i \lambda_{a}} \hat{\Phi}_{a}$ for each component and

$$
\left(\hat{\Phi}_{a}, \hat{\Phi}_{b}\right) \rightarrow\left(\hat{\Phi}_{a} e^{i \lambda_{a b} x^{b}}, \hat{\Phi}_{b} e^{-i \lambda_{a b} x^{a}}\right)
$$

$$
\hat{\Phi}=\left(\begin{array}{l}
\odot \\
\odot
\end{array}\right)
$$

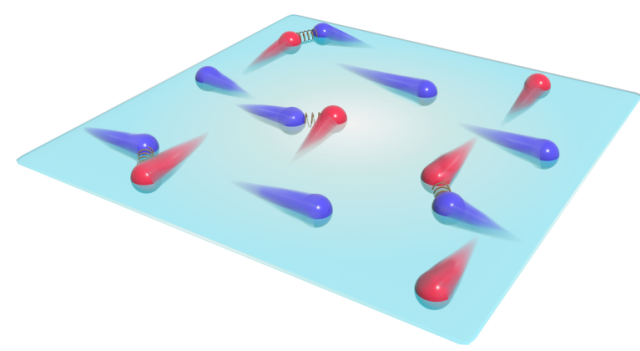

FIG. 1. Illustration of the interacting system in two dimensions. The red and blue balls respectively represent two components $a=$ 1,2, which move along distinct orthogonal directions. The spring between two balls represents the interaction due to the $K$-term in Eq. (1).

for each pair of indices with $\lambda_{a}, \lambda_{a b} \in \mathbb{R}$. The parameters $\lambda_{a b}$ are antisymmetric $\lambda_{a b}=-\lambda_{b a}$, thus inducing $\frac{d(d-1)}{2}$ independent conserved angular charge moments $Q_{a b}$. The transformations in Eq. (4) involve local coordinates $\mathbf{x}=$ $\left(x^{1}, x^{2}, \ldots, x^{d}\right)$, and they do not form an internal symmetry. We denote the symmetry group as $\mathcal{G}$ which characterizes a higher-rank symmetry [65]. In a periodic boundary condition, the parameters $\lambda_{a b}$ have the dimension of $[x]^{-1}$ and quantization of the related charges is expected to coincide with a momentum. This symmetry intertwines global and internal symmetries such that strong constraints are imposed on particles' propagations. In two dimensions, conservation of $Q_{12}=\int d^{2} x\left(\hat{\rho}_{1} x^{2}-\hat{\rho}_{2} x^{1}\right)$ requires the velocity shall be parallel to a vector $\left(\hat{\rho}_{1}, \hat{\rho}_{2}\right)$ as a lineon. In three spatial dimensions, we have three angular charge moments $Q_{12}, Q_{23}, Q_{13}$, such that a particle only propagates in the direction parallel to $\left(\hat{\rho}_{1}, \hat{\rho}_{2}, \hat{\rho}_{3}\right)$. Generally, fundamental particles in $d \mathrm{D}$ move with velocity parallel to $\left(\hat{\rho}_{1}, \ldots, \hat{\rho}_{d}\right)$. Figure 1 pictorially shows the interacting system when $d=2$.

\section{B. Hartree-Fock-Bogoliubov mean-field theory: condensate and rotons}

It is well known that a Bose-Einstein condensate consists of a two-component structure: the condensate and the normal components. The HFB mean-field theory allows factorization of fields $\hat{\Phi}_{a}$ in terms of an appropriate orthonormal singleparticle basis,

$$
\hat{\Phi}_{a}(\mathbf{x})=\phi_{a 0}(\mathbf{x}) \hat{c}_{a 0}+\sum_{i \neq 0} \phi_{a i}(\mathbf{x}) \hat{c}_{a i} \equiv \phi_{a 0}(\mathbf{x}) \hat{c}_{a 0}+\hat{\psi}_{a}(\mathbf{x}),
$$

with $\hat{\psi}_{a}(\mathbf{x}) \equiv \sum_{i \neq 0} \phi_{a i}(\mathbf{x}) \hat{c}_{a i}$ where the operator $\hat{c}_{a i}$ satisfies the bosonic commutation relations $\left[\hat{c}_{a i}, \hat{c}_{b j}^{\dagger}\right]=\delta_{a b} \delta_{i j}$. The wave functions $\phi_{a 0}(\mathbf{x})$ signify the condensate component. The noncondensate fields $\hat{c}_{a i}(i \geqslant 1)$ constitute a branch of gapped quasiparticle excitations and are orthogonal with the condensate component $\phi_{a 0}(\mathbf{x})$,

$$
\int d^{d} \mathbf{x} \phi_{a 0}(\mathbf{x}) \phi_{b i}^{*}(\mathbf{x})=0 \text { for } i \geqslant 1 .
$$


Here the subscript index " $i$ " can be taken as a spectral index of the BdG Hamiltonian [see Eq. (16)], and thus $\phi_{a i}$ can denote wave functions of low-energy excitations. We take the normal component as a perturbation to the condensate. Substituting Eq. (5) into the Hamiltonian in Eq. (1) leads to a partition into terms with different numbers of field operators $\hat{\psi}_{a}(\mathbf{x})$. The zeroth-order term is given by

$$
H_{0}=\sum_{a=1}^{d} h_{1, a} \hat{N}_{a 0}+\sum_{a, b} h_{2, a b}\left(\hat{N}_{a 0} \hat{N}_{b 0}-\delta_{a b} \hat{N}_{a 0}\right),
$$

where $\hat{N}_{a 0}=\hat{c}_{a 0}^{\dagger} \hat{c}_{a 0}$ is the number operator for the condensate and $h_{1}\left(h_{2}\right)$ is the expectation value of the single-particle (twoparticle) part of the Hamiltonian,

$$
\begin{aligned}
h_{1, a}= & \int d^{d} \mathbf{x}\left|\partial_{a} \phi_{a 0}\right|^{2}-\mu\left|\phi_{a 0}\right|^{2} \\
h_{2, a b}= & \int d^{d} \mathbf{x} \frac{1}{2} K_{a b}\left|\phi_{a 0} \partial_{a} \phi_{b 0}+\phi_{b 0} \partial_{b} \phi_{a 0}\right|^{2} \\
& +\frac{g \delta_{a b}}{2}\left|\phi_{a 0} \phi_{b 0}\right|^{2} .
\end{aligned}
$$

Under the particle-number representation $\left|\left\{N_{a 0}\right\}\right\rangle$ with definite condensate particle number $\hat{N}_{b 0}\left|\left\{N_{a 0}\right\}\right\rangle=N_{b 0}\left|\left\{N_{a 0}\right\}\right\rangle$ for $b=$ $1, \ldots, d$, the ground state energy $E_{0}\left(\left\{N_{a}\right\}\right)$ of $H_{0}$ in Eq. (7) only depends on the condensate,

$$
\begin{aligned}
E_{0}\left(\left\{N_{a}\right\}\right) & =\left\langle\left\{N_{a 0}\right\}\left|H_{0}\right|\left\{N_{a 0}\right\}\right\rangle \\
& =\sum_{a} h_{1, a} N_{a 0}+\sum_{a, b} h_{2, a b}\left(N_{a 0} N_{b 0}-\delta_{a b} N_{a 0}\right) .
\end{aligned}
$$

The next order $H_{1}$ has linear dependence on $\hat{\psi}_{a}(\mathbf{x})$,

$$
H_{1}=\int d^{d} \mathbf{x} \sum_{a=1}^{d} \hat{c}_{a 0}^{\dagger} \phi_{a 0}^{*} \hat{\mathcal{H}}_{a}^{\dagger} \hat{\psi}_{a}+\hat{\psi}_{a}^{\dagger} \hat{\mathcal{H}}_{a} \phi_{a 0} \hat{c}_{a 0},
$$

where

$$
\hat{\mathcal{H}}_{a}=-\partial_{a}^{2}-\mu+g\left|\phi_{a 0}\right|^{2} \hat{N}_{a 0}+\sum_{a, b} \frac{1}{2} K_{a b} \hat{N}_{b 0} \hat{\mathcal{H}}_{a b},
$$

with

$$
\begin{aligned}
\hat{\mathcal{H}}_{a b}= & \left(\partial_{a} \phi_{b 0}^{*}\right)\left(\partial_{a} \phi_{b 0}\right)+\left(\partial_{a} \phi_{b 0}^{*}\right) \phi_{b 0} \partial_{b}-\left(\partial_{b} \phi_{b 0}^{*}\right)\left(\partial_{a} \phi_{b 0}\right) \\
& -\phi_{b 0}^{*}\left(\partial_{a} \phi_{b 0}\right) \partial_{b}-\phi_{b 0}^{*}\left(\partial_{a} \partial_{b} \phi_{b 0}\right)-\left(\partial_{b} \phi_{b 0}^{*}\right) \phi_{b 0} \partial_{b} \\
& -\phi_{b 0}^{*}\left(\partial_{b} \phi_{b 0}\right) \partial_{b}-\phi_{b 0}^{*} \phi_{b 0} \partial_{b}^{2} .
\end{aligned}
$$

Under a basis $\left|\left\{N_{a 0}\right\}\right\rangle$, taking the limit of $N_{a 0} \gg 1$, one can recognize $\hat{N}_{a 0} \hat{c}_{a 0}\left|\left\{N_{a}\right\}\right\rangle=\hat{c}_{a 0} \hat{N}_{a 0}\left|\left\{N_{a}\right\}\right\rangle$. If $\phi_{a 0}(\mathbf{x})$ are chosen to be eigenstates of the operator $\hat{\mathcal{H}}_{a}$ in Eq. (12),

$$
\hat{\mathcal{H}}_{a}(\mathbf{x}) \phi_{a 0}(\mathbf{x})=\epsilon_{a 0}\left(\left\{N_{a 0}\right\}\right) \phi_{a 0}(\mathbf{x}) a=1, \ldots d,
$$

then $H_{1}$ vanishes identically due to orthogonality in Eq. (6) and $N_{a 0} \gg 1$, where ground state energy $\epsilon_{a 0}\left(\left\{N_{a 0}\right\}\right)$ merely depends on the condensate component. This fact establishes the validity of the expansion in Eq. (5). Equation (13) marks a set of the GP equations describing the condensate components where $\hat{\mathcal{H}}_{a}$ in Eq. (12) behaves as a single-particle Hamiltonian. It simply directs us to approximate the original Hamiltonian in Eq. (1) by Eq. (7). With the translational symmetry, the GP equations have a set of very simple solutions. If the chemical potential $\mu$ is negative, the ground state energy $E_{0}$ reaches its minimum when the condensate has vanishing density, $N_{a 0}=0$ for $a=1, \ldots d$. We obtain a normal state. If the chemical potential is switched to a positive value, the ground state energy $E_{0}$ reaches maximum with a finite number of the condensate component. In this case, the configurations of ground states can be parametrized by real parameters $\theta_{a}$ and $\beta_{a b}\left(\beta_{a b}=-\beta_{b a}, a, b=1, \ldots d\right)$,

$$
\phi_{a 0}(\mathbf{x})=\frac{1}{\sqrt{V}} e^{i\left(\theta_{a}+\sum_{b=1}^{d} \beta_{a b} x^{b}\right)},
$$

with $V$ as the spatial volume. Remarkably, Eq. (14) depends on the parameters $\beta_{a b}$ which carry the dimension of "momentum." In other words, the condensate component can carry finite momentum. Of course, one can include the trap potential that can break a translational symmetry, under which the GP equations may be short of analytical solutions. Casting the solution in Eq. (14) back to $H_{0}$ in Eq. (7), we have the ground state energy $E_{0}\left(\left\{N_{a 0}\right\}\right)$,

$$
E_{0}\left(\left\{N_{a 0}\right\}\right)=\sum_{a=1}^{d}-\mu \frac{N_{a 0}}{V}+\frac{g}{2}\left(\frac{N_{a 0}}{V}\right)^{2} .
$$

The minimal condition of $E_{0}\left(\left\{N_{a 0}\right\}\right)$ with a positive chemical potential $\mu$ requires $\rho_{a 0} \equiv \frac{N_{a 0}}{V}=\frac{\mu}{g}$, which indicates the ground states for a fractonic superfluid has a macroscopically finite particle density. Hence, we obtain a superfluid phase by condensing lineons, which we dub $d \mathrm{SF}^{1}$. Therefore, select one ground state in Eq. (14) and we can fix the condensate particle number $N_{a 0}$ by simply replacing both $\hat{c}_{a 0}$ and $\hat{c}_{a 0}^{\dagger}$ operators by c-number $\sqrt{N_{a 0}}$, which indicates occurrence of ODLRO with the condensate density $\rho_{a 0}$.

The next order $\mathrm{H}_{2}$ goes beyond the GP equation to include the quadratic terms of $\psi_{a}$,

$$
\begin{aligned}
H_{2}= & \sum_{a=1}^{d} \int d^{d} \mathbf{x} \hat{\psi}_{a}^{\dagger}\left(-\partial_{a}^{2}+g \rho_{a 0}\right) \hat{\psi}_{a} \\
& +\sum_{a, b} \frac{1}{2} K_{a b} \rho_{a 0}\left(\partial_{a} \hat{\psi}_{b}^{\dagger}+\partial_{b} \hat{\psi}_{a}^{\dagger}\right)\left(\partial_{a} \hat{\psi}_{b}+\partial_{b} \hat{\psi}_{a}\right) \\
& +\sum_{a=1}^{d} \frac{g}{2} \rho_{a 0}\left(\hat{\psi}_{a}^{\dagger} \hat{\psi}_{a}^{\dagger}+\hat{\psi}_{a} \hat{\psi}_{a}\right)
\end{aligned}
$$

where we have replaced $\hat{c}_{a 0}$ and $\hat{c}_{a 0}^{\dagger}$ with $\sqrt{N_{a 0}}$. New quadratic terms emerge from the $K_{a b}$ term which relaxes the restrictions on dynamics. It means that the quasiparticle modes $\hat{\psi}_{a}$ can propagate along all spatial directions. The mass term $g \rho_{a 0} \hat{\psi}_{a}^{\dagger} \hat{\psi}_{a}$ originates from the condensate component. $H_{2}$ in Eq. (16) is designated as a BdG Hamiltonian to characterize the noncondensate quasiparticle modes. One can diagonalize $\mathrm{H}_{2}$ to obtain the canonical quaisparticle modes. For example, in $2 \mathrm{D}$, the spectrum has two branches,

$$
\epsilon_{ \pm}=\sqrt{\left[\frac{\left(1+K \rho_{0}\right)|\mathbf{k}|^{2}}{2} \pm \frac{\Delta(\mathbf{k})}{2}+g \rho_{0}\right]^{2}-\left(g \rho_{0}\right)^{2}} .
$$



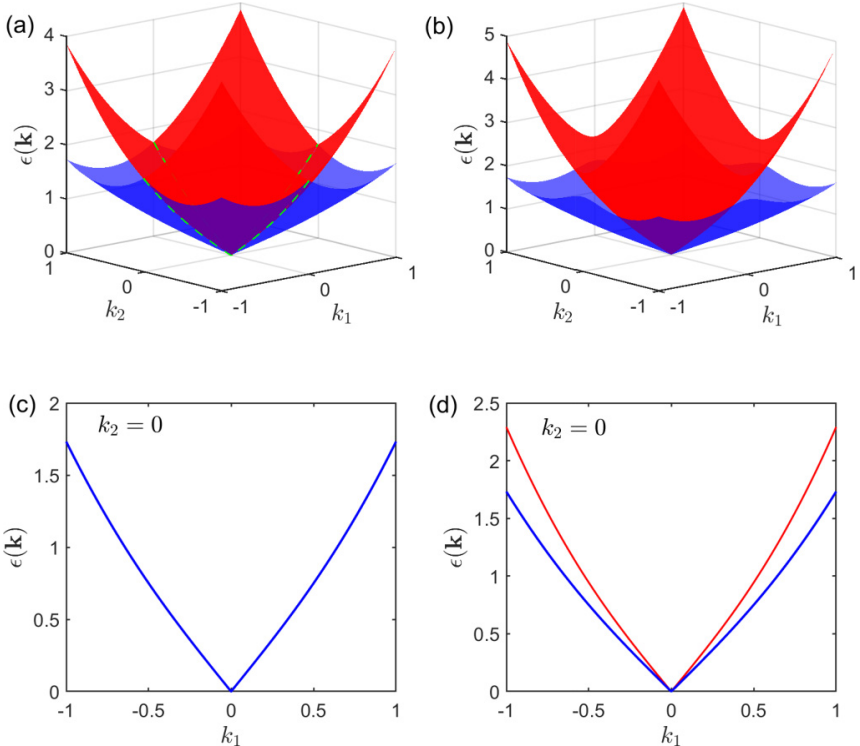

FIG. 2. Spectrum dispersions of quasiparticles from HFB approximation in Eq. (17) in 2D with parameters in (a) and (c) $K=$ $1, \rho_{0}=1$, and (b) and (d) $K=1.5, \rho_{0}=1$. In (a) the two bands are degenerate at two lines $k_{1}=0$ and $k_{2}=0$ which are guided by green dashed lines. The gap between the two bands in (b) keeps finite except at the point $\mathbf{k}=\mathbf{0}$. (c) and (d) show the dispersion relations at the line $k_{2}=0$. Both in (a) and (b), the dispersions are linear at small $\mathbf{k}$ and quadratic at large $\mathbf{k}$.

For small momentum, up to the first order, we have linear gapless dispersion relations,

$$
\epsilon_{ \pm}(\mathbf{k})=\sqrt{g \rho_{0}} \sqrt{\left(1+K \rho_{0}\right) \mathbf{k}^{2} \pm \Delta(\mathbf{k})} \text { small } \mathbf{k},
$$

and they describe two gapless phonon excitations, while at the large momentum, spectrum dispersions depend on momentum quadratically,

$$
\epsilon_{ \pm}=\frac{\left(1+K \rho_{0}\right)|\mathbf{k}|^{2}}{2} \pm \frac{\Delta(\mathbf{k})}{2} \quad \text { large } \mathbf{k}
$$

and instead they correspond to gapped roton modes. Here $\Delta(\mathbf{k}) \equiv \sqrt{\left(k_{1}^{2}-k_{2}^{2}\right)^{2}\left(K \rho_{0}-1\right)^{2}+4 K^{2} \rho_{0}^{2} k_{1}^{2} k_{2}^{2}}, \rho_{0}=\frac{\mu}{g}$, and $K \equiv K_{12}$. The smooth change from linear to quartic dispersion is the key feature of the HFB approximation. Although two modes, phonons and rotons, are emphasized, indeed they represent different behaviors at small and high momentum, respectively. The splitting between the two dispersion relations in Eqs. (18) and (19) is controlled by $\Delta(\mathbf{k})$. If $K \rho_{0}=1$ such that $\Delta(\mathbf{k})=0$, the two bands are degenerate at two lines $k_{1}=0$ or $k_{2}=0$ and along the two lines, the bands have ill-defined curvatures, which is expected to be detected by thermal Hall effect. Figure 2 depicts the two dispersion relations in Eq. (17) in which the two cases of $K \rho_{0}=1$ or not.

Higher-order terms couple the normal with the condensate part and describe the interaction between phonon modes, which is beyond the scope of this work and we leave it to future work.

\section{EFFECTIVE FIELD THEORY}

The HFB mean-field method unifies gapless phonon modes and gapped roton modes via a BdG Hamiltonian in Eq. (16) in a fractonic superfluid phase $d \mathrm{SF}^{1}$. Nevertheless, gapless mode excitations can destroy BEC or ODLRO. In this section, we deal with gapless modes of $d \mathrm{SF}^{1}$ in the framework of a continuous field theory and discuss stability of $d \mathrm{SF}^{1}$ against quantum fluctuations.

\section{A. Euler-Lagrange equation and Noether charge/current}

For the coherence and completeness of the present section, we re-derive some quantities from the field-theoretical perspective.

We perform a coherent-state path integral quantization [68] to get the Lagrangian density $\mathcal{L}$,

$$
\mathcal{L}=\sum_{a=1}^{d} i \phi_{a}^{*} \partial_{t} \phi_{a}-\mathcal{H}(\phi),
$$

where $\phi_{a}(\mathbf{x}, t)$ is an eigenvalue of $\hat{\Phi}_{a}(\mathbf{x})$ on a coherent state $\hat{\Phi}_{a}(\mathbf{x})\left|\phi_{a}(\mathbf{x}, t)\right\rangle=\phi_{a}(\mathbf{x}, t)\left|\phi_{a}(\mathbf{x}, t)\right\rangle$. The first-order derivative in Eq. (20) in nature is determined by the commutation relation in Eq. (2) which can be confirmed through the canonical quantization. For convenience, we apply the Wick's rotation to an imaginary time at zero temperature $T=0$.

Next, we can derive the Euler-Lagrange equations as well as the Noether currents associated with two types of conserved quantities. The Euler-Lagrange equations can be derived from the formula $\frac{\delta \mathcal{L}}{\delta \phi_{a}^{*}}=0$, explicitly, $i \partial_{t} \phi_{a}=\hat{H}_{a} \phi_{a}(a=1, \ldots, d)$, where $\hat{H}_{a}$ has the same form as $\mathcal{H}_{a}$ in Eq. (12),

$$
\hat{H}_{a}=-\partial_{a}^{2}-\mu+g\left|\phi_{a}\right|^{2}+\frac{1}{2} \sum_{b} \mathcal{H}_{a b} .
$$

Here $\mathcal{H}_{a b}$ comes from the $K_{a b}$ term,

$$
\begin{aligned}
\mathcal{H}_{a b}= & K_{a b} \partial_{a} \phi_{b}^{*} \partial_{a} \phi_{b}+K_{a b} \partial_{a} \phi_{b}^{*} \phi_{b} \partial_{b}-K_{a b} \partial_{b} \phi_{b}^{*} \partial_{a} \phi_{b} \\
& -K_{a b} \phi_{b}^{*} \partial_{a} \phi_{b} \partial_{b}-K_{a b} \phi_{b}^{*} \partial_{a} \partial_{b} \phi_{b}-K_{a b} \partial_{b} \phi_{b}^{*} \phi_{b} \partial_{b} \\
& -K_{a b} \phi_{b}^{*} \partial_{b} \phi_{b} \partial_{b}-K_{a b} \phi_{b}^{*} \phi_{b} \partial_{b}^{2} .
\end{aligned}
$$

The Euler-Lagrange equations just recover the GP equations. Here $\phi_{a}$ plays the same role of representing the condensate component as $\phi_{a 0}$ in Eq. (13).

The Hamiltonian in Eq. (1) stays invariant under transformation in Eq. (4) as well as the particle number conservation symmetry. For the infinitesimal change $\delta \phi_{a}=i \alpha_{a} \phi_{a}$, we have the related Noether charge $Q_{a}$ with charge density $\rho_{a}$ and currents $J_{i}^{a}$ that read

$$
\begin{aligned}
Q^{a}= & \int d^{d} \mathbf{x} \phi_{a}^{*} \phi_{a} \equiv \int d^{d} \mathbf{x} \rho_{a}, \\
J_{i}^{a}= & i K_{a i} \rho_{a}\left(\phi_{i} \partial_{a} \phi_{i}^{*}-\phi_{i}^{*} \partial_{a} \phi_{i}\right) \\
& +i K_{a i} \rho_{i}\left(\phi_{a} \partial_{i} \phi_{a}^{*}-\phi_{a}^{*} \partial_{i} \phi_{a}\right) \\
& +i\left(\phi_{i} \partial_{i} \phi_{i}^{*}-\phi_{i}^{*} \partial_{i} \phi_{i}\right) \delta_{a i},
\end{aligned}
$$

which satisfies the continuity equations $\partial_{t} \rho^{a}+\sum_{i} \partial_{i} J_{i}^{a}=$ 0 . Here in Eq. (23), coincidence between $\phi_{a}^{*} \phi_{a}$ and particle density $\rho_{a}$ arises from the first-order time derivative in the Hamiltonian in Eq. (1). For the transformation $\delta \phi_{a}=$ 
$i x_{b} \phi_{a}$ and $\delta \phi_{b}=-i x_{a} \phi_{b}$ corresponding to Eq. (4), we have conserved angular moments $Q_{a b}$ (with density $\rho_{a b}$ ) and currents $D_{i}^{a b}$,

$$
\begin{aligned}
Q_{a b} & =\int d^{d} x\left(\rho_{a} x^{b}-\rho_{b} x^{a}\right) \equiv \int d^{d} x \rho_{a b}, \\
D_{i}^{a b} & =x^{b} J_{i}^{a}-x^{a} J_{i}^{b},
\end{aligned}
$$

with $\rho_{a}$ and $J_{i}^{a}$ as $U(1)$ charge and current in Eqs. (23) and (24). The continuity equation $\partial_{t} \rho^{a b}+\sum_{i=1}^{d} \partial_{i} D_{i}^{a b}=0$ is automatically satisfied as long as the currents $J_{b}^{a}$ obey the relations $J_{b}^{a}=J_{a}^{b}$.

\section{B. Goldstone modes and quantum fluctuations}

The HFB mean-field theory in Sec. II B starts with one of the classical field configurations $\phi_{a}^{\mathrm{cl}}=\sqrt{\rho_{0}} e^{\left(i \theta_{a}+i \sum_{b=1}^{d} \beta_{a b} x^{b}\right)}$ which can be formulated in the second quantization language as

$$
|\mathrm{GS}\rangle_{\theta_{a}}^{\beta_{a b}}=\prod_{a=1}^{d} \exp \left[\sqrt{\rho_{0}} e^{i\left(\theta_{a}+\sum_{b=1}^{d} \beta_{a b} x^{b}\right)} \hat{\Phi}_{a}^{\dagger}(\mathbf{x})\right]|0\rangle,
$$

where $\hat{\Phi}_{a}^{\dagger}(\mathbf{x})(a=1, \ldots, d)$ creates an $a$ th-component lineon with restricted motion. The salience of Eq. (27) features a finite expectation value of operator $\hat{\Phi}_{a}(\mathbf{x})$,

$$
\left\langle\mathrm{GS}\left|\hat{\Phi}_{a}(\mathbf{x})\right| \mathrm{GS}\right\rangle_{\theta_{a}}^{\beta_{a b}}=\sqrt{\rho_{0}} \exp \left(i \theta_{a}+i \sum_{b} \beta_{a b} x^{b}\right),
$$

thus marking an ODLRO and we obtain a fractonic superfluid phase $d \mathrm{SF}^{1}$. The expectation value oscillates as a plane wave with fixed momentum $\mathbf{k}_{a}=\left(\beta_{a 1}, \beta_{a 2}, \ldots, \beta_{a d}\right)$ for the $a$-component particle. In this sense, we can rewrite $|\mathrm{GS}\rangle_{\theta_{a}}^{\beta_{a b}}=$ $\prod_{a=1}^{d} \exp \left[\sqrt{\rho_{0}} e^{i \theta_{a}} \hat{\Phi}_{a}^{\dagger}\left(\mathbf{k}_{a}\right)\right]|0\rangle$ with $\hat{\Phi}_{a}^{\dagger}\left(\mathbf{k}_{a}\right)$ being the Fourier transformation of $\hat{\Phi}_{a}^{\dagger}(\mathbf{x})$. These features arise from restricted mobility of condensed particles. As a side note, there is an interesting observation. The ground state in Eq. (27), which carries a finite momentum, looks like an Fulde-Ferrell-LarkinOvchinnikov (FFLO) state [69,70], if we define the FFLO state as an ordered phase described by an order parameter with a finite momentum. But there are fundamental differences in the microscopic mechanisms. The general FFLO states in the literature are formed by Cooper pairs of fermions (i.e., electrons) and the momentum of the center of mass of a Cooper pair is nonzero. In contrast, the superfluid phase in Eq. (27) is directly formed by condensing multicomponent bosonic lineons. The degenerate ground-state subspace of fractonic superfluids is generated by group elements of higher-rank symmetry group $\mathcal{G}$ (see $\mathrm{Sec}$. II A), thereby spontaneously breaking the higher-rank symmetry group. In other words, the microscopic Hamiltonian of fractonic superfluids must respect an exotic higher-rank symmetry. Despite the differences, it still will be valuable to explore the possibility of realizing fractonic superfluids from a more microscopic model where "molecules" formed by fermion pairings can be regarded as bosonic lineons.

After condensation, the Noether currents in Eqs. (24) and (26) reduce to simpler forms by expanding the field $\phi_{a}=$ $\phi_{a}^{\mathrm{cl}} e^{i \theta_{a}}$ where $\phi_{a}^{\mathrm{cl}}$ denotes classical configurations and $\theta_{a}$ are the quantum phase fluctuations,

$$
\rho_{a}=\rho_{0}, J_{i}^{a}=2 K \rho_{0}^{2}\left(\partial_{i} \theta_{a}+\partial_{a} \theta_{i}\right)+2 \rho_{0} \partial_{i} \theta_{a} \delta_{a i},
$$

and

$$
\rho_{a b}=\rho_{0}\left(x^{b}-x^{a}\right), D_{i}^{a b}=x^{b} J_{i}^{a}-x^{a} J_{i}^{b} .
$$

To derive the effective theory for quantum fluctuations or the gapless Goldstone modes, we expand the fields around a selected classical configuration $\phi_{a}(\mathbf{x}, t)=$ $\sqrt{\rho_{0}+\rho_{a}(\mathbf{x}, t)} e^{i \theta_{a}(\mathbf{x}, t)}$ where $\rho_{a}$ and $\theta_{a}$ denote density and phase fluctuations, respectively. Up to the second order, we have

$$
\begin{aligned}
\mathcal{L}= & \sum_{a=1}^{d}-\rho_{a} \partial_{t} \theta_{a}-\rho_{0}\left(\partial_{a} \theta_{a}\right)^{2}-\frac{1}{4 \rho_{0}}\left(\partial_{a} \rho_{a}\right)^{2}-\frac{g}{2} \rho_{a}^{2} \\
& -\sum_{a, b} \frac{1}{2} K_{a b} \rho_{0}^{2}\left(\partial_{a} \theta_{b}+\partial_{b} \theta_{a}\right)^{2} \\
& -\frac{1}{8} \sum_{a, b} K_{a b}\left(\partial_{a} \rho_{b}+\partial_{b} \rho_{a}\right)^{2} .
\end{aligned}
$$

The density fluctuation fields $\rho_{a}(a=1, \ldots, d)$ work as auxiliary fields that are subject to the constraint equations,

$$
\begin{aligned}
\partial_{t} \theta_{a}= & -g \rho_{a}-\left(\frac{1}{2 \rho_{0}} \partial_{a}^{2}+\frac{1}{2} \sum_{b} K_{a b} \partial_{b}^{2}\right) \rho_{a} \\
& -\frac{1}{2} \sum_{b} K_{a b} \partial_{a} \partial_{b} \rho_{b} .
\end{aligned}
$$

Since we are only interested in the low-energy physics, the momentum $\mathbf{k}$ has an upper bound $|\mathbf{k}| \leqslant 2 \pi \xi_{c}^{-1}$ where the coherent length $\xi_{c}$ can be estimated as

$$
g=4 \pi^{2} \xi_{c}^{-2}\left(\frac{1}{2 \rho_{0}}+\sum_{b} K_{a b}\right)
$$

as such the last two terms in Eq. (32) can be neglected. In the low-energy limit, we obtain the solutions $\rho_{a}=-\frac{1}{g} \partial_{t} \theta_{a}$. Cast it back to Eq. (31) and we arrive at an effective theory of quantum fluctuations by excluding the higher derivative terms of $\theta_{a}$,

$$
\begin{aligned}
\mathcal{L}= & \sum_{a} \frac{1}{2 g}\left(\partial_{t} \theta_{a}\right)^{2}-\rho_{0}\left(\partial_{a} \theta_{a}\right)^{2} \\
& -\sum_{a, b} \frac{1}{2} K_{a b} \rho_{0}^{2}\left(\partial_{a} \theta_{b}+\partial_{b} \theta_{a}\right)^{2} .
\end{aligned}
$$

The effective theory in Eq. (34) stays invariant under the transformation $\theta_{a} \rightarrow \theta_{a}+\lambda_{a}+\sum_{b} \lambda_{a b} x^{b}$ with $\lambda_{a b}=-\lambda_{b a}$. It describes $d$ gapless Goldstone modes $\theta_{a}(a=1, \ldots, d)$ [71] that have entangled motions arising from the $K_{a b}$ term.

To get a deeper insight, we concentrate ourselves on the two-dimensional case. Introduce the canonical modes $\Theta_{+}(\mathbf{k})$ and $\Theta_{-}(\mathbf{k})$,

$$
\begin{gathered}
\Theta_{+}(\mathbf{k})=\cos \frac{\varphi_{\mathbf{k}}}{2} \theta_{1}(\mathbf{k})+\sin \frac{\varphi_{\mathbf{k}}}{2} \theta_{2}(\mathbf{k}), \\
\Theta_{-}(\mathbf{k})=-\sin \frac{\varphi_{\mathbf{k}}}{2} \theta_{1}(\mathbf{k})+\cos \frac{\varphi_{\mathbf{k}}}{2} \theta_{2}(\mathbf{k}),
\end{gathered}
$$


where $\tan \frac{\varphi_{\mathbf{k}}}{2}=\frac{2 K \rho_{0} k_{1} k_{2}}{\Delta(\mathbf{k})^{2}} \quad$ with denoting $K_{12}=K \quad$ and their dispersion relations take the form $\epsilon_{ \pm}(\mathbf{k})=$ $\sqrt{g \rho_{0}\left[\left(1+K \rho_{0}\right) \mathbf{k}^{2} \pm \Delta(\mathbf{k})\right]}$, which is identical to Eq. (18).

Stability of a superfluid phase is determined by the longdistance behavior of the correlator of the order parameter under the influence of quantum fluctuations,

$$
\begin{aligned}
& \left\langle\mathrm{GS}\left|\Phi_{a}^{\dagger}(\mathbf{x}) \Phi_{b}(\mathbf{0})\right| \mathrm{GS}\right\rangle_{\theta_{a}}^{\beta_{a b}} \\
& \quad=\rho_{0} \exp \left[i \sum_{c}\left(\beta_{b c}-\beta_{a c}\right) x^{c}\right]\left\langle e^{-i \Theta_{a}(\mathbf{x})} e^{i \Theta_{b}(\mathbf{0})}\right\rangle .
\end{aligned}
$$

We need to calculate equal-time correlators of the canonical modes $\left\langle e^{-i \Theta_{ \pm}(\mathbf{x})} e^{i \Theta_{ \pm}(\mathbf{0})}\right\rangle=e^{-\frac{1}{2}\left\langle\left[\Theta_{ \pm}(\mathbf{x})-\Theta_{ \pm}(\mathbf{0})\right]^{2}\right\rangle}$. Explicitly, in two spatial dimensions, we have

$$
\begin{aligned}
\left\langle\Theta_{ \pm}(\mathbf{x}) \Theta_{ \pm}(\mathbf{0})\right\rangle & =\int \frac{d \omega d^{2} k}{(2 \pi)^{3}} \frac{e^{i \mathbf{k} \cdot \mathbf{x}}}{\omega^{2}-\omega_{ \pm}(\mathbf{k})} \\
& =\int \frac{d k d \theta}{(2 \pi)^{2}} \frac{e^{i k|\mathbf{x}| \cos \theta}}{\sqrt{g \rho_{0}\left[\left(1+K \rho_{0}\right) \pm \bar{\Delta}(\theta)\right]}} \\
& <\int \frac{d k d \theta}{(2 \pi)^{2}} \frac{e^{i k|\mathbf{x}| \cos \theta}}{\sqrt{g \rho_{0} c_{ \pm}}}=\frac{1}{2 \pi|\mathbf{x}|} \frac{1}{\sqrt{g \rho_{0} c_{ \pm}}},
\end{aligned}
$$

where $\bar{\Delta}(\theta)=\sqrt{\left(1-2 K \rho_{0}\right) \cos ^{2} 2 \theta+K^{2} \rho_{0}^{2}}$ and $c_{ \pm}$denotes the minimum value of $\left(1+K \rho_{0}\right) \pm \bar{\Delta}(\theta)$.

At the long distance $|\mathbf{x}| \rightarrow \infty$, the correlator $\left\langle\Theta_{ \pm}(\mathbf{x}) \Theta_{ \pm}(\mathbf{0})\right\rangle \quad$ vanishes. Thus, $\left\langle\Phi_{a}^{\dagger}(\mathbf{x}) \Phi_{b}(\mathbf{0})\right\rangle=$ $\rho_{0} \exp \left[i \sum_{c}\left(\beta_{b c}-\beta_{a c}\right) x^{c}\right]$ has a finite value modulated by a plane wave. It confirms a true long-range order that survives against quantum fluctuations when both the two lineons condensate simultaneously in zero temperature. Since quantum fluctuations are weaker in higher dimensions, a fractonic superfluid phase $d \mathrm{SF}^{1}$ stays stable in two spatial dimensions $d=2$ and higher $d>2$. In Appendix A, the component-dependent Mexican-hat potential in 2D is considered, which leads to condensation of only one component of lineons. The uncondensed component gets released from mobility restriction and the Goldstone mode behaves still as a subdimensional particle. Thus, the superfluid there obeys an algebraic order.

\section{SUPERFLUID VORTICES OF 2 SF $^{1}$}

Besides the gapless Goldstone modes and gapped roton modes, thermal vortices are fundamental to a superfluid phase as an effect of compactness of phase fields $\theta_{a}$. The existence of symmetry in Eq. (4) admits a complicated structure in $2 \mathrm{SF}^{1}$. We present two guiding statements on construction of point thermal vortices in 2D and then give the two types of vortices in $2 \mathrm{SF}^{1}$

\section{A. Two statements on construction}

A superfluid vortex is an excitation as a consequence of compactness of a phase field and mathematically one can represent compactness by a multivalued function. Given a phase field $\theta_{a}$ with component $a$, we can always decompose it as $\theta_{a}(\mathbf{x})=\theta_{a}^{v}(\mathbf{x})+\theta_{a}^{s}(\mathbf{x})$ where $\theta_{a}^{s}(\mathbf{x})$ denotes the smooth component. In general, the multivalued component $\theta_{a}^{v}(\mathbf{x})$ can be formulated as

$$
\theta_{a}^{v}(\mathbf{x})=f_{a}(\mathbf{x}) \varphi(\mathbf{x})
$$

where $\varphi(\mathbf{x})$ defined mod $2 \pi$ is the angle of site $\mathbf{x}$ relative to vortex core and $f_{a}(\mathbf{x})$ is a single-valued function. Equation (39) sets an equivalent relation $\theta_{a}^{v}(\mathbf{x}) \sim \theta_{a}^{v}(\mathbf{x})+2 \pi f_{a}(\mathbf{x})$. Subtly, $f_{a}(\mathbf{x})$ should be understood under a lattice regularization to protect single valuedness of field $\phi_{a}$, where spatial coordinates $\mathbf{x}$ are regarded as $\mathbf{x}=\left(x^{1}, x^{2}\right)=\mathbf{n} a$ with $\mathbf{n}=$ $\left(n_{1}, n_{2}\right)$ being a pair of integers and $a$ being the lattice constant. The equivalence relation in Eq. (39) resembles a gauge freedom. Whether we start with $\theta_{a}^{v}(\mathbf{x})$ or $\theta_{a}^{v}(\mathbf{x})+2 \pi f_{a}(\mathbf{x})$ should cause no physical effects. Therefore, we arrive at Statement 1 below.

Statement 1 . The physical Hamiltonian density should be single valued even in the presence of multivalued vortex configurations.

Statement 1 clarifies that the Hamiltonian density $\mathcal{H}\left[\theta_{a}(\mathbf{x})\right]$ is invariant when $\theta_{a}(\mathbf{x})$ is shifted by $2 \pi f_{a}(\mathbf{x}), \mathcal{H}\left[\theta_{a}(\mathbf{x})+\right.$ $\left.2 \pi f_{a}(\mathbf{x})\right]=\mathcal{H}\left[\theta_{a}(\mathbf{x})\right]$, which determines the most singular part of a vortex. Take a conventional superfluid $2 \mathrm{SF}^{2}$ as an example with Hamiltonian density $\mathcal{H}=\frac{1}{2}\left[\left(\partial_{1} \theta(\mathbf{x})\right)^{2}+\right.$ $\left.\left(\partial_{2} \theta(\mathbf{x})\right)^{2}\right]$. With the assumption $\theta^{v}(\mathbf{x})=f(\mathbf{x}) \varphi(\mathbf{x})$, the constraint imposed by Statement 1 on shifting $\theta(\mathbf{x})$ by $2 \pi f(\mathbf{x})$ gives the equations $\partial_{1} f(\mathbf{x})=0, \partial_{2} f(\mathbf{x})=0$ towards which we have the solution $f(\mathbf{x})=\ell$ with $\ell \in \mathbb{Z}$. Thus we recover vortex configurations in a conventional superfluid.

The second statement to be introduced below controls the smooth component after we obtain the multivalued component from Statement 1. A higher-rank symmetry group contains not only conventional $U(1)$ charges that induce a global $U(1)$ phase shift, but also charges that generate a phase shift depending on local coordinates. For convenience, we call conventional $U(1)$ charges as rank-0 while the others are higher-rank charges. Statement 2 below establishes the relations between higher-rank and rank-0 charges.

Statement 2. The action of a higher-rank symmetry group on some bound states of operators charged in the higher-rank symmetry group is equivalent to an action of a global $U(1)$ symmetry with appropriate rank-0 charges.

Statement 2 allows us to construct a set of bound states such that the higher-rank group only induces a global phase shift. Explicitly, given vortices carrying higher-rank charges, Statement 2 claims that some bound state of these vortices is proportional to $\varphi(\mathbf{x})$ as a conventional vortex, that is, the smooth component vanishes. Thus, the essence is to find the structures of bound states which are significantly determined by relations between higher-rank charges and rank- 0 charges. For example, we consider a higher-rank symmetry [47] which shifts $\theta(\mathbf{x})$ by $\theta(\mathbf{x}) \rightarrow \theta(\mathbf{x})+\lambda+\sum_{a} \lambda_{a} x^{a}$. Then the group action on bound states like $\hat{\mathcal{O}}_{\mathbf{d}}=e^{-i \theta(\mathbf{x})} e^{i \theta(\mathbf{x}-\mathbf{d})}$ with a constant vector $\mathbf{d}$ generates a pure global phase, $\hat{\mathcal{O}}_{\mathbf{d}} \rightarrow$ $\hat{\mathcal{O}}_{\mathbf{d}} e^{-i \sum_{a} \lambda_{a} d^{a}}$. Thus, on these bound states, the higher-rank group is equivalent to group $U(1)$ and we shall expect that $\hat{\mathcal{O}}_{\mathbf{d}}$ takes the form of a conventional vortex whose smooth part can be set to vanish. In Appendix B, we present a detailed derivation on vortices in a higher-rank superfluid phase in Ref. [47]. 


\section{B. Vortex structure}

The two statements are more generally applicable for a system of a higher-rank symmetry. At present, we specialize our attention to the case of $2 \mathrm{SF}^{1}$. Statement 1 leads to an assumption for the multivalued component,

$$
\theta_{1}^{v}(\mathbf{x})=f_{1}(\mathbf{x}) \varphi(\mathbf{x}), \theta_{2}^{v}(\mathbf{x})=f_{2}(\mathbf{x}) \varphi(\mathbf{x}),
$$

and $f_{1,2}(\mathbf{x})$ should satisfy the following equations:

$$
\partial_{1} f_{1}(\mathbf{x})=0, \partial_{2} f_{2}(\mathbf{x})=0, \partial_{2} f_{1}(\mathbf{x})+\partial_{1} f_{2}(\mathbf{x})=0 .
$$

The solutions generally can be parametrized by three parameters,

$$
f_{1}(\mathbf{x})=p x^{2}+\ell_{1}, f_{2}(\mathbf{x})=-p x^{1}+\ell_{2} .
$$

Here, $p$ has the dimension $[x]^{-1}$, which we dub a dipole charge, while $\ell_{1}$ and $\ell_{2}$ are dimensionless. Under the lattice regularization, $p a$ and $\ell_{1}, \ell_{2}$ are all integers.

The parameters $\ell_{1}$ and $\ell_{2}$ describe conventional vortices and they are interpreted as the winding numbers. To obtain vortices carrying a dipole charge $p$, we apply Statement 2 . The essence of Statement 2 is to recognize bound states. In $2 \mathrm{D}$, the higher-rank group $\mathcal{G}$ is parametrized by $\lambda_{1}, \lambda_{2}$, and $\lambda_{12}$. We denote the group element with $\lambda_{1}=\lambda_{2}=0, \lambda_{12}=1$ as $G_{e}$. Given a vortex operator $\hat{\mathcal{O}}_{1}(\mathbf{x})=e^{i \theta_{1}(\mathbf{x})}$ with the charge $-q_{12}$, which is transformed by $G_{e}$ in $\mathcal{G}$ as $\hat{\mathcal{O}}_{1}(\mathbf{x}) \rightarrow \hat{\mathcal{O}}_{1}(\mathbf{x}) e^{i q_{12} x^{2}}$, then a "particle-hole" bound state $\hat{\mathcal{O}}_{1}^{\dagger}(\mathbf{x}) \hat{\mathcal{O}}_{1}(\mathbf{x}-\mathbf{d})$ with a constant vector $\mathbf{d}=(0, d)$ is transformed by $G_{e}$ as

$$
\hat{\mathcal{O}}_{1}^{\dagger}(\mathbf{x}) \hat{\mathcal{O}}_{1}(\mathbf{x}-\mathbf{d}) \rightarrow \hat{\mathcal{O}}_{1}^{\dagger}(\mathbf{x}) \hat{\mathcal{O}}_{1}(\mathbf{x}-\mathbf{d}) e^{-i q_{12} d} .
$$

If the particle-hole bound state is attributed with a $U(1)$ charge $q_{12} d$, we find the action in Eq. (43) can be re-explained as the action of $U(1)$ symmetry. Statement 2 asserts that the bound state reduces to a conventional vortex, which imposes a constraint $\theta_{1}(\mathbf{x})-\theta_{1}(\mathbf{x}-\mathbf{d})=d \partial_{2} \theta_{1}(\mathbf{x})=q_{12} d \varphi(\mathbf{x})$ for small $\mathbf{d}$. We have

$$
\begin{gathered}
p d \varphi(\mathbf{x})-q_{12} d \varphi(\mathbf{x})=0, \\
\partial_{2} \theta_{1}^{s}(\mathbf{x})+f_{1}(\mathbf{x}) \partial_{2} \varphi(\mathbf{x})=0 .
\end{gathered}
$$

Equation (44) shows $p=q_{12}$ which indicates the dipole charge $p$ in $f_{1}(\mathbf{x})$ represents a higher-rank charge of group $\mathcal{G}$. In fact, the bound state with $\mathbf{d}=(d, 0)$ is invariant under $G_{e}$ and thus it requires $\hat{\mathcal{O}}_{1}^{\dagger}(\mathbf{x}) \hat{\mathcal{O}}_{1}(\mathbf{x}-\mathbf{d})$ to be single valued, which is satisfied since $f_{1}(\mathbf{x})$ is independent of $x^{1}$. We consider vortex operator object $\hat{\mathcal{O}}_{2}(\mathbf{x})=e^{i \theta_{2}}$ carrying a charge $q_{12}$ with a transformation by $G_{e}$ as $\hat{\mathcal{O}}_{2}(\mathbf{x}) \rightarrow \hat{\mathcal{O}}_{2}(\mathbf{x}) e^{-i q_{12} x^{1}}$. Then $G_{e}$ induces a global phase shift on the bound state $\hat{\mathcal{O}}_{2}(\mathbf{x})^{\dagger} \hat{\mathcal{O}}_{2}(\mathbf{x}-\mathbf{d}) \rightarrow \hat{\mathcal{O}}_{2}(\mathbf{x})^{\dagger} \hat{\mathcal{O}}_{2}(\mathbf{x}-\mathbf{d}) e^{i q_{12} d}$ with $\mathbf{d}=(d, 0)$. Thus, we are allowed to re-interpret action of $\mathcal{G}$ on a bound state $\hat{\mathcal{O}}_{2}(\mathbf{x})^{\dagger} \hat{\mathcal{O}}_{2}(\mathbf{x}-\mathbf{d})$ as an action of the $U(1)$ group on a charged $-q_{12} d$ operator. Therefore, according to Statement 2, we have $\theta_{2}(\mathbf{x})-\theta_{2}(\mathbf{x}-\mathbf{d})=-q_{12} d \varphi(\mathbf{x})$ for small $d$. Equivalently, we have

$$
\begin{aligned}
& -p d \varphi(\mathbf{x})+q_{12} d \varphi(\mathbf{x})=0, \\
& \partial_{1} \theta_{2}^{s}(\mathbf{x})+f_{2}(\mathbf{x}) \partial_{1} \varphi(\mathbf{x})=0 .
\end{aligned}
$$

Here the dipole charge denotes a higher-rank charge of group $\mathcal{G}$.
Thus, we can obtain two types of vortices. The first one is the conventional vortex characterized by winding numbers $\ell_{1}, \ell_{2}$,

$$
\theta_{1}(\mathbf{x})=\ell_{1} \varphi(\mathbf{x}), \quad \theta_{2}(\mathbf{x})=\ell_{2} \varphi(\mathbf{x}) .
$$

And the second one with a configuration,

$$
\begin{aligned}
& \theta_{1}(\mathbf{x})=-p x^{1} \log |\mathbf{x}|+p x^{2} \varphi(\mathbf{x}), \\
& \theta_{2}(\mathbf{x})=-p x^{2} \log |\mathbf{x}|-p x^{1} \varphi(\mathbf{x}),
\end{aligned}
$$

carries a higher-rank charge $p$ of symmetry $\mathcal{G}$. We emphasize again that the charge $p$ should be regularized as $p=$ $\ell a^{-1}(\ell \in \mathbb{Z})$ to ensure $f_{1,2}(\mathbf{x})$ in Eq. (40) to be integer valued. When we circle around the vortex core, the vortex configuration gets an extra phase $\delta \theta_{1}=2 \pi p x^{2}=2 \pi \ell n_{2}$ and $\delta \theta_{2}=-2 \pi p x=-2 \pi \ell n_{1}$ with $\mathbf{x}=\left(n_{1}, n_{2}\right) a$, which keeps in consistence with compactness of $\theta_{a}$. Different from a conventional vortex, here $\partial_{1} \theta_{2}$ and $\partial_{2} \theta_{1}$ are still multivalued while $\partial_{1} \theta_{1}$ and $\partial_{2} \theta_{2}$ are single valued.

We can define the vorticity for the dipole charge by recombination of Noether currents. After condensation, in two spatial dimensions the Noether currents can be formulated as

$$
J_{1}^{1}=2 \rho_{0} \partial_{1} \theta_{1}, J_{2}^{2}=2 \rho_{0} \partial_{2} \theta_{2}, J_{1}^{2}=2 K \rho_{0}^{2}\left(\partial_{1} \theta_{2}+\partial_{2} \theta_{1}\right) .
$$

As indicated by Statement 2, a "particle-hole" bound state of vortices behaves as a vortex in $2 \mathrm{SF}^{2}$ and it only encodes the dipole charge. Above all, the density $\rho_{\text {dipole }}$ of such a bound state can be written as

$$
\rho_{\text {dipole }}=\sum_{a, b=1}^{2} \frac{1}{2} \epsilon_{a b} \partial_{a} \partial_{b}\left(\partial_{2} \theta_{1}-\partial_{1} \theta_{2}\right),
$$

where $\epsilon_{a b}$ is an antisymmetric tensor $\epsilon_{12}=-\epsilon_{21}=1$. Actually we have a relation,

$$
\rho_{\text {dipole }}=\sum_{a, b=1}^{2} \epsilon_{a b} \partial_{a} \partial_{b} \partial_{2} \theta_{1}=\sum_{a, b=1}^{2}-\epsilon_{a b} \partial_{a} \partial_{b} \partial_{1} \theta_{2},
$$

since $\theta_{1}$ and $\theta_{2}$ take the same dipole charge. Following the lesson we learned for vortices in superfluid phase $2 \mathrm{SF}^{2}$, we can construct the currents $\mathbf{J}_{\text {dipole }}$ based on condensed currents in Eq. (51) with components,

$$
\begin{gathered}
\mathbf{J}_{\text {dipole }}^{1}=-\frac{1}{2} a K^{-1} \rho_{0}^{-2} \partial_{1} J_{1}^{2}+a \rho_{0}^{-1} \partial_{2} J_{1}^{1}, \\
\mathbf{J}_{\text {dipole }}^{2}=\frac{1}{2} a K^{-1} \rho_{0}^{-2} \partial_{2} J_{1}^{2}-a \rho_{0}^{-1} \partial_{1} J_{2}^{2},
\end{gathered}
$$

where the cutoff $a$ is introduced to make up the dimension of $\mathbf{J}_{\text {dipole }}$. Then the vorticity will give the dipole charge,

$$
\ell_{\text {dipole }}=\frac{1}{2 \pi} \oint_{C} d \mathbf{x} \cdot \mathbf{J}_{\text {dipole }}=\ell,
$$

where $C$ is a closed path encircling the vortex core and $p=\ell a^{-1}$.

\section{CONCLUDING REMARKS}

As a series of the work [47], we have further explored more possibilities of exotic states of matter formed by particles with restricted mobility. We have discussed a fractonic superfluid phase $d \mathrm{SF}^{1}$ in a microscopic model by condensing subdimensional particles. This model is invariant under 
a higher-rank symmetry such that its fundamental particles are lineons. We use the HFB mean-field theory to derive a set of highly nonlinear GP equations and a BdG Hamiltonian which characterize the condensed and the norm components, respectively. In the framework of a continuous field theory, we construct macroscopic degeneracies of ground states with finite momentum and derive an effective theory for gapless Goldstone modes. At zero temperature, a phase $d \mathrm{SF}^{1}$ stays stable in two spatial dimensions and higher. We emphasize two guiding statements to construct vortex excitations in two spatial dimensions. Explicitly, there are two types of vortices in $2 \mathrm{SF}^{1}$. Besides conventional vortices, the other type carries a dipole charge. The two guiding statements are more generally applicable (see Appendix B).

Towards a complete understanding of a fractonic superfluid phase, we have to deal with more questions. Tightly related to the present paper, vortex excitations form a hierarchy which is dominated by the two statements, and then interactions between vortices and BKT transitions, should also inherit such a hierarchy. A natural question is to investigate a superfluid phase by condensing other spatially extended excitations [27]. On the other hand, by viewing the model in Eq. (1) as coupled Luttinger liquids, we can derive an effective theory by bosonization. Then, it is straightforward to obtain universal properties of fractonic superfluids such as conductivity and general thermodynamic properties [72,73]. In three spatial dimensions, more exotic vortex line excitations can be excited and their construction needs further investigation. If we condense these defects to recover the symmetry as the scheme to construct a symmetry-protected topological phase [74-78], what phase can be obtained? Besides, what is the universal class of the phase transition between a high-rank superfluid phase and a normal state? Experimentally, we expect that the Hamiltonian in Eq. (1) can be realized in the cold atomic gas subjected to an optical lattice by tuning a two-particle state [79], which opens a new horizon to search exotic phases of matter.

\section{ACKNOWLEDGMENTS}

We thank Yuxuan Wang, Zhi Wang, Wen Huang, and JianHua Jiang for their useful discussions. This work was supported in part by the Sun Yat-sen University startup grant, Guangdong Basic and Applied Basic Research Foundation under Grant No. 2020B1515120100, and National Natural Science Foundation of China (NSFC) (Grants No. 11847608 and No. 12074438).

\section{APPENDIX A: COMPONENT-DEPENDENT POTENTIAL}

In the main text, a Mexican-hat potential is chosen to be component independent and the two components of lineons are condensed simultaneously. Here, we briefly discuss a component-dependent Mexican-hat potential in two spatial dimensions with only one of the two components condensated. In the framework of coherent-path integral representation, we introduce a Mexican-hat potential only for $\phi_{2}$,

$$
V=-\mu_{2}\left|\phi_{2}\right|^{2}+\frac{g_{2}}{2}\left|\phi_{2}\right|^{4}
$$

with $g_{2}>0$. When $\mu_{2}<0, \phi_{2}$ is in an insulating state. When $\mu_{2}>0, \phi_{2}$ picks up a finite particle density, $\rho_{20} \equiv \frac{\mu_{2}}{g_{2}}$ to minimize the potential in Eq. (A1). In the classical level, the configurations of $\phi_{2}$ take the form as

$$
\phi_{2}^{\mathrm{cl}}=\sqrt{\rho_{20}} e^{i \theta_{2}+i \beta_{12} x^{2}} .
$$

We can expand the field $\phi_{2}$ around $\phi_{2}^{\mathrm{cl}}$ in Eq. (A2). The obtained Hamiltonian explicitly depends on the parameter $\beta_{12}$. This dependence can be removed by a transformation,

$$
\phi_{1}(\mathbf{x}) \rightarrow \phi_{1}(\mathbf{x}) e^{-i \beta_{12} x^{1}} .
$$

Following the procedure in Sec. III and up to second order, we obtain an effective theory,

$$
\begin{aligned}
\mathcal{L}\left[\phi_{1}\right] & =i \phi_{1}^{*} \partial_{t} \phi_{1}-\left|\partial_{1} \phi_{1}\right|^{2}-K \rho_{20}\left|\partial_{2} \phi_{1}\right|^{2}, \\
\mathcal{L}\left[\theta_{2}\right] & =\frac{1}{2 g_{2}}\left(\partial_{t} \theta_{2}\right)^{2}-\rho_{20}\left(\partial_{2} \theta_{2}\right)^{2},
\end{aligned}
$$

where $K=K_{12}$. The symmetry of $\phi_{1}$ in Eq. (A4) reduces to a conventional particle conservation $U(1)$ symmetry after $\phi_{2}$ condensation, and thus the field $\phi_{1}$ gets liberated from the mobility restriction due to the last term in Eq. (A4). However, the Goldstone mode $\theta_{2}$ still lacks full mobility and can only propagate in one direction. The stability can be inferred from the long-range behaviors of order parameter correlator $\left\langle e^{i \theta_{2}(\mathbf{x})} e^{-i \theta_{2}(\mathbf{0})}\right\rangle=e^{-\frac{1}{2}\left\langle\left(\theta_{2}(\mathbf{x})-\theta_{2}(\mathbf{0})\right)^{2}\right\rangle}$. which is determined by

$$
\left\langle\theta_{2}(\mathbf{x}) \theta_{2}(\mathbf{0})\right\rangle=\delta\left(x^{1}\right) \int \frac{d \omega d k_{2}}{(2 \pi)^{3}} e^{-i k_{2} x^{2}} \frac{g_{2}}{\omega^{2}-\mu_{2} k_{2}^{2}} .
$$

If $x^{1} \neq 0$, the correlator in Eq. (A6) vanishes as a natural consequence of mobility constraint. When $x^{1}=0$, we have

$$
\left\langle\theta_{2}(\mathbf{x}) \theta_{2}(\mathbf{0})\right\rangle=-\frac{2 \pi}{\xi} \frac{\pi g_{2}}{\sqrt{\mu_{2}}} \log \frac{4 e^{-\gamma}\left(x^{2}\right)^{2}}{\mu_{2}},
$$

such that $\left\langle e^{i \theta_{2}(\mathbf{x})} e^{-i \theta_{2}(\mathbf{0})}\right\rangle$ decays in a power-law pattern where $\xi$ is the coherent length and $\gamma$ is the Euler constant. Therefore, the superfluid phase here is algebraically ordered.

\section{APPENDIX B: APPLICATION OF THE TWO STATEMENTS}

In the main body, we put forward two statements to construct vortex excitations and indicate they are generally applicable. Here, we apply them to a fracton model that is considered in Ref. [47]. The Hamiltonian density for the Goldstone modes reads

$$
\mathcal{H}=\frac{1}{2}\left[\left(\partial_{1}^{2} \theta\right)^{2}+2\left(\partial_{1} \partial_{2} \theta\right)^{2}+\left(\partial_{2}^{2} \theta\right)^{2}\right] .
$$

The vortex excitations arise when $\theta$ is multivalued. We decompose the vortex field as $\theta=\theta^{v}+\theta^{s}$, where the multivalued component can be formulated as

$$
\theta^{v}(\mathbf{x})=f(\mathbf{x}) \varphi(\mathbf{x}),
$$

with $\varphi(\mathbf{x})$ the angle of site $\mathbf{x}=\left(x^{1}, x^{2}\right)$ relative to the vortex core. Statement 1 requires Hamiltonian density $\mathcal{H}$ be single valued for the field configuration in Eq. (B2), which induces the restrictions on $f(\mathbf{x})$,

$$
\partial_{1} f(\mathbf{x})=0, \quad \partial_{2} f(\mathbf{x})=0 .
$$


It is easy to find the solutions,

$$
f(\mathbf{x})=\ell,
$$

or

$$
f(\mathbf{x})=p_{1} x^{1}, \quad f(\mathbf{x})=p_{2} x^{2} .
$$

The first solution in Eq. (B4) marks a conventional vortex solution with $\theta=\ell \varphi(\mathbf{x})$ where $\ell \in \mathbb{Z}$ represents the winding number. The second solution in Eq. (B5) takes charges $p_{1}, p_{2}$ with dimension $\left[x^{-1}\right]$. The charges are expected to be quantized as a momentum. The full solution should take into consideration the smooth component $\theta^{s}(\mathbf{x})$, which is instructed by Statement 2. For $f(\mathbf{x})=p_{1} x^{1}$, we consider a bound state of a vortex-anti-vortex pair with distance $\mathbf{d}=$ $(d, 0)$, on which the vortex bound state reduces to a conventional vortex with winding number $d q_{1}$, i.e.,

$$
d \partial_{1}\left[p_{1} x^{1} \varphi(\mathbf{x})+\theta^{s}(\mathbf{x})\right]=d q_{1} \varphi(\mathbf{x}) .
$$

So $\theta^{s}(\mathbf{x})=q_{1} x^{2} \log |\mathbf{x}|$. Similarly, consider a bound state of a vortex-anti-vortex pair with distance $\mathbf{d}=(0, d)$ and we can obtain the smooth component for $f(\mathbf{x})=-q_{2} x \log |\mathbf{x}|$. In summary, vortices for the model in Eq. (B1) have the configurations as

$$
\begin{gathered}
\theta=\ell \varphi(\mathbf{x}), \\
\theta=p_{1} x^{1} \varphi(\mathbf{x})+p_{1} y \log |\mathbf{x}|, \\
\theta=p_{2} x^{2} \varphi(\mathbf{x})-p_{2} x \log |\mathbf{x}| .
\end{gathered}
$$

[1] C. Chamon, Quantum Glassiness in Strongly Correlated Clean Systems: An Example of Topological Overprotection, Phys. Rev. Lett. 94, 040402 (2005).

[2] J. Haah, Local stabilizer codes in three dimensions without string logical operators, Phys. Rev. A 83, 042330 (2011).

[3] S. Vijay, J. Haah, and L. Fu, A new kind of topological quantum order: A dimensional hierarchy of quasiparticles built from stationary excitations, Phys. Rev. B 92, 235136 (2015).

[4] S. Vijay, J. Haah, and L. Fu, Fracton topological order, generalized lattice gauge theory, and duality, Phys. Rev. B 94, 235157 (2016).

[5] A. Prem, J. Haah, and R. Nandkishore, Glassy quantum dynamics in translation invariant fracton models, Phys. Rev. B 95, 155133 (2017).

[6] W. Shirley, K. Slagle, and X. Chen, Foliated fracton order from gauging subsystem symmetries, Sci. Post. Phys. 6, 41 (2019).

[7] H. Ma, E. Lake, X. Chen, and M. Hermele, Fracton topological order via coupled layers, Phys. Rev. B 95, 245126 (2017).

[8] D. Bulmash and M. Barkeshli, Gauging fractons: Immobile non-Abelian quasiparticles, fractals, and position-dependent degeneracies, Phys. Rev. B 100, 155146 (2019).

[9] A. Prem and D. Williamson, Gauging permutation symmetries as a route to non-Abelian fractons, Sci. Post. Phys. 7, 068 (2019).

[10] D. Bulmash and M. Barkeshli, Generalized $U(1)$ gauge field theories and fractal dynamics, arXiv:1806.01855.

[11] K. T. Tian, E. Samperton, and Z. Wang, Haah codes on general three-manifolds, Ann. Phys. 412, 168014 (2020).

[12] Y. You, D. Litinski, and F. von Oppen, Higher-order topological superconductors as generators of quantum codes, Phys. Rev. B 100, 054513 (2019)

[13] H. Ma, M. Hermele, and X. Chen, Fracton topological order from the Higgs and partial-confinement mechanisms of ranktwo gauge theory, Phys. Rev. B 98, 035111 (2018).

[14] K. Slagle and Y. B. Kim, Fracton topological order from nearest-neighbor two-spin interactions and dualities, Phys. Rev. B 96, 165106 (2017).
[15] G. B. Halász, T. H. Hsieh, and L. Balents, Fracton Topological Phases from Strongly Coupled Spin Chains, Phys. Rev. Lett. 119, 257202 (2017).

[16] K. T. Tian and Z. Wang, Generalized haah codes and fracton models, arXiv: 1902.04543 .

[17] W. Shirley, K. Slagle, and X. Chen, Fractional excitations in foliated fracton phases, Ann. Phys. 410, 167922 (2019).

[18] K. Slagle, D. Aasen, and D. Williamson, Foliated field theory and string-membrane-net condensation picture of fracton order, Sci. Post. Phys. 6, 43 (2019).

[19] W. Shirley, K. Slagle, Z. Wang, and X. Chen, Fracton Models on General Three-Dimensional Manifolds, Phys. Rev. X 8, 031051 (2018).

[20] A. Prem, S.-J. Huang, H. Song, and M. Hermele, Cage-Net Fracton Models, Phys. Rev. X 9, 021010 (2019).

[21] S. Pai, M. Pretko, and R. M. Nandkishore, Localization in Fractonic Random Circuits, Phys. Rev. X 9, 021003 (2019).

[22] S. Pai and M. Pretko, Dynamical Scar States in Driven Fracton Systems, Phys. Rev. Lett. 123, 136401 (2019).

[23] P. Sala, T. Rakovszky, R. Verresen, M. Knap, and F. Pollmann, Ergodicity Breaking Arising from Hilbert Space Fragmentation in Dipole-Conserving Hamiltonians, Phys. Rev. X 10, 011047 (2020).

[24] A. Kumar and A. C. Potter, Symmetry-enforced fractonicity and two-dimensional quantum crystal melting, Phys. Rev. B 100, 045119 (2019).

[25] M. Pretko, The fracton gauge principle, Phys. Rev. B 98 115134 (2018).

[26] M. Pretko, Subdimensional particle structure of higher rank $u$ (1) spin liquids, Phys. Rev. B 95, 115139 (2017).

[27] M.-Y. Li and P. Ye, Fracton physics of spatially extended excitations, Phys. Rev. B 101, 245134 (2020).

[28] M. Pretko, Generalized electromagnetism of subdimensional particles: A spin liquid story, Phys. Rev. B 96, 035119 (2017).

[29] L. Radzihovsky and M. Hermele, Fractons from Vector Gauge Theory, Phys. Rev. Lett. 124, 050402 (2020).

[30] A. Dua, I. H. Kim, M. Cheng, and D. J. Williamson, Sorting topological stabilizer models in three dimensions, Phys. Rev. B 100, 155137 (2019). 
[31] A. Gromov, Chiral Topological Elasticity and Fracton Order, Phys. Rev. Lett. 122, 076403 (2019).

[32] J. Haah, Lattice quantum codes and exotic topological phases of matter, Ph.D thesis, California Institute of Technology, 2013.

[33] A. Gromov, Towards Classification of Fracton Phases: The Multipole Algebra, Phys. Rev. X 9, 031035 (2019).

[34] Y. You, T. Devakul, S. L. Sondhi, and F. J. Burnell, Fractonic Chern-Simons and BF theories, Phys. Rev. Res. 2, 023249 (2020).

[35] J. Sous and M. Pretko, Fractons from polarons and hole-doped antiferromagnets: Microscopic realizations, Phys. Rev. B 102, 214437 (2020).

[36] V. Khemani, M. Hermele, and R. Nandkishore, Localization from Hilbert space shattering: From theory to physical realizations, Phys. Rev. B 101, 174204 (2020).

[37] J. Wang and K. Xu, Higher-rank tensor field theory of nonabelian fracton and embeddon, Annals of Physics 424, 168370 (2021).

[38] J. Wang and S.-T. Yau, Non-Abelian gauged fracton matter field theory: Sigma models, superfluids, and vortices, Phys. Rev. Res. 2, 043219 (2020)

[39] S. Pai and M. Pretko, Fractonic line excitations: An inroad from three-dimensional elasticity theory, Phys. Rev. B 97, 235102 (2018).

[40] M. Pretko and R. M. Nandkishore, Localization of extended quantum objects, Phys. Rev. B 98, 134301 (2018).

[41] D. J. Williamson, Z. Bi, and M. Cheng, Fractonic matter in symmetry-enriched $u(1)$ gauge theory, Phys. Rev. B 100, 125150 (2019).

[42] A. Dua, D. J. Williamson, J. Haah, and M. Cheng, Compactifying fracton stabilizer models, Phys. Rev. B 99, 245135 (2019).

[43] B. Shi and Y.-M. Lu, Deciphering the nonlocal entanglement entropy of fracton topological orders, Phys. Rev. B 97, 144106 (2018).

[44] H. Song, A. Prem, S.-J. Huang, and M. A. Martin-Delgado, Twisted fracton models in three dimensions, Phys. Rev. B 99, 155118 (2019).

[45] H. Ma and M. Pretko, Higher-rank deconfined quantum criticality at the Lifshitz transition and the exciton Bose condensate, Phys. Rev. B 98, 125105 (2018).

[46] J. Wang, K. Xu, and S.-T. Yau, Higher-rank tensor non-Abelian field theory: Higher-moment or subdimensional polynomial global symmetry, algebraic variety, Noether's theorem, and gauging, Phys. Rev. Research 3, 013185 (2021).

[47] J.-K. Yuan, S. A. Chen, and P. Ye, Fractonic superfluids, Phys. Rev. Research 2, 023267 (2020).

[48] K. Slagle, Foliated quantum field theory of fracton order, arXiv:2008.03852.

[49] D. J. Williamson and T. Devakul, Type-II fractons from coupled spin chains and layers, arXiv:2007.07894.

[50] P. Gorantla, H. Tat Lam, N. Seiberg, and S.-H. Shao, More exotic field theories in 3+1 dimensions, SciPost Phys. 9, 073 (2020).

[51] D. X. Nguyen, A. Gromov, and S. Moroz, Fracton-elasticity duality of two-dimensional superfluid vortex crystals: defect interactions and quantum melting, SciPost Phys. 9, 076 (2020).

[52] M. Pretko, S. A. Parameswaran, and M. Hermele, Odd fracton theories, proximate orders, and parton constructions, Phys. Rev. B 102, 205106 (2020).
[53] D. J. Williamson and M. Cheng, Designer non-Abelian fractons from topological layers, arXiv:2004.07251.

[54] N. Seiberg and S.-H. Shao, Exotic $\mathbb{Z}_{N}$ symmetries, duality, and fractons in 3+1-dimensional quantum field theory, SciPost Phys. 10, 003 (2021).

[55] D. T. Stephen, J. Garre-Rubio, A. Dua, and D. J. Williamson, Subsystem symmetry enriched topological order in three dimensions, Phys. Rev. Res. 2, 033331 (2020).

[56] N. Seiberg and S.-H. Shao, Exotic $U(1)$ symmetries, duality, and fractons in 3+1-dimensional quantum field theory, Sci. Post Phys. 9, 46 (2020).

[57] A. Gromov, A. Lucas, and R. M. Nandkishore, Fracton hydrodynamics, Phys. Rev. Res. 2, 033124 (2020).

[58] J. Wang, Non-liquid cellular states, arXiv:2002.12932.

[59] W. Shirley, Fractonic order and emergent fermionic gauge theory, arXiv:2002.12026.

[60] D. Aasen, D. Bulmash, A. Prem, K. Slagle, and D. J. Williamson, Topological defect networks for fractons of all types, Phys. Rev. Research 2, 043165 (2020).

[61] X.-G. Wen, A systematic construction of gapped non-liquid states, Phys. Rev. Research 2, 033300 (2020).

[62] T. F. J. Poon and X.-J. Liu, Quantum phase transition of fracton topological orders, arXiv:2001.05937.

[63] R. M. Nandkishore and M. Hermele, Fractons, Annu. Rev. Condens. Matter Phys. 10, 295 (2019).

[64] M. Pretko, X. Chen, and Y. You, Fracton phases of matter, Int. J. Mod. Phys. A 35, 2030003 (2020).

[65] N. Seiberg, Field theories with a vector global symmetry, Sci. Post Phys. 8, 050 (2020).

[66] C. N. Yang, Concept of off-diagonal long-range order and the quantum phases of liquid he and of superconductors, Rev. Mod. Phys. 34, 694 (1962).

[67] A. L. Fetter, J. D. Walecka, B. Banes, and M. Eakens, Quantum Theory of Many-particle Systems, International series in pure and applied physics (McGraw-Hill, New York, 1971).

[68] A. Altland and B. D. Simons, Condensed Matter Field Theory, 2nd ed. (Cambridge University Press, Cambridge, 2010).

[69] P. Fulde and R. A. Ferrell, Superconductivity in a strong spinexchange field, Phys. Rev. 135, A550 (1964).

[70] A. I. Larkin and Y. N. Ovchinnikov, Nonuniform state of superconductors, Zh. Eksp. Teor. Fiz. 47, 1136 (1964) [Sov. Phys. JETP 20, 762 (1965)].

[71] H. Watanabe, Counting rules of Nambu-Goldstone modes, Annu. Rev. Condens. Matter Phys. 11, 169 (2020).

[72] A. Paramekanti, L. Balents, and M. P. Fisher, Ring exchange, the exciton Bose liquid, and bosonization in two dimensions, Phys. Rev. B 66, 054526 (2002).

[73] J. Sullivan, A. Dua, and M. Cheng, Fractonic topological phases from coupled wires, arXiv:2010.15148.

[74] X. Chen, Y.-M. Lu, and A. Vishwanath, Symmetry-protected topological phases from decorated domain walls, Nat. Commun. 5, 3507 (2014).

[75] Z.-C. Gu, J. C. Wang, and Xiao-Gang Wen, Multikink topological terms and charge-binding domain-wall condensation induced symmetry-protected topological states: Beyond Chern-Simons/BF field theories, Phys. Rev. B 93, 115136 (2016). 
[76] P. Ye and Z.-C. Gu, Topological quantum field theory of threedimensional bosonic Abelian-symmetry-protected topological phases, Phys. Rev. B 93, 205157 (2016).

[77] P. Ye and Z.-C. Gu, Vortex-Line Condensation in Three Dimensions: A Physical Mechanism for Bosonic Topological Insulators, Phys. Rev. X 5, 021029 (2015).

[78] AtMa P. O. Chan, P. Ye, and S. Ryu, Braiding with Borromean rings in $(3+1)$-Dimensional Spacetime, Phys. Rev. Lett. 121, 061601 (2018).
[79] H. P. Büchler, M. Hermele, S. D. Huber, M. P. Fisher, and P. Zoller, Atomic Quantum Simulator for Lattice Gauge Theories and Ring Exchange Models, Phys. Rev. Lett. 95, 040402 (2005).

Correction: The name of a funding source listed in the Acknowledgment section contained an error and has been corrected. 